\title{
Relation between Aggregation Behavior of Glutenin and Its Polypeptide Composition
}

\author{
Tsutomu AraKawa, Minoru Yoshida, Hideaki Morishita, \\ Junko HondA and Daizo Yonezawa \\ Laboratory of Food Chemistry, College of Agriculture, University \\ of Osaka Prefecture, Sakai, Japan \\ Received December 28, 1976
}

\begin{abstract}
It was found in the previous studies that the quality of flour is closely related to the aggregation behavior of gluten separated from it, and that difference of aggregation behavior of gluten was mainly due to the nature of glutenin contained in it. In the present paper, aggregation behaviors of glutenins were investigated in relation to their polypeptide compositions with ten kinds of flours. Component polypeptides of glutenin were fractionated into three fractions, FI, FII and FIII, by gel filtration, and their polypeptide compositions and aggregation behaviors were investigated. The ratio of the three fractions was different among the ten glutenins, and a correlation was found between $\tau_{10} / \mathrm{C}$ values, a parameter for aggregation reaction, of glutenins and their FI and FII contents. $\tau_{10} / \mathrm{C}$ decreased with increasing content of FI and with decreasing content of FII. Therefore, the aggregation behavior of gluten depends at least partly on the contents of FI and FII in glutenin.
\end{abstract}

The quality of wheat flour is dependent not only on the quantity of gluten but also on its quality. ${ }^{1 \sim 4)}$ Smith and Mullen $^{5)}$ investigated the dispersibility of gluten in the mixing dough with two different flours in quality, and found a difference in dispersibility between them. They ${ }^{6}$ also observed that addition of reagents such as sodium dodecyl sulfate changed the dispersibility of gluten as well as the mixing property of flour. These findings suggest that the mixing property of flour is dependent on the gluten dispersibility in dough mixing. To find the cause of the difference of gluten dispersibility among flours, we investigated the aggregation behavior of gluten, instead of its dispersibility, ${ }^{7)}$ and found that the aggregation behavior of gluten was different depending on the flour from which it was separated and that there was a correlation between the quality of flour and the aggregation behavior of gluten. ${ }^{8}$ It was also shown that the difference of aggregation behavior of gluten was mainly due to the nature of glutenin contained in it. ${ }^{8,9)}$ These results lead us to an investigation on what makes the difference of aggregation behavior of glutenin among the flours. In the present paper, component polypeptides of glutenin were fractionated into three fractions, FI, FII and FIII, by gel filtration and relation between the aggregation behavior of glutenin and its FI, FII and FIII content was investigated with ten kinds of wheat flours.

\section{MATERIALS AND METHODS}

Wheat flours. Flours used were ten kinds of unbleached flours listed in Table II. They were the same flours as used in the previous paper. ${ }^{8)}$

Preparation of glutens. Glutens were separated from flours according to the method of Jones et al. ${ }^{10)}$

Preparation of glutenins. Glutens were fractionated into glutenins and gliadins by gel filtration on a Sephadex G-100 column $(5 \times 100 \mathrm{~cm})$ in $0.1 \mathrm{~m}$ acetic acid, as described in the previous paper..

Fractionation of component polypeptides of glutenin by gel filtration. Glutenins were reduced and cyanoethylated with 2-mercaptoethanol and acrylonitrile as described in the previous paper.9) After cyanoethylation, the reaction mixture was acidified and dialyzed against $0.1 \mathrm{M}$ acetic acid. Component polypeptides thus obtained were fractionated into three fractions, FI, FII and FIII, by gel filtration on a Sephadex G-150 column $(4 \times 100 \mathrm{~cm})$ in $0.1 \mathrm{M}$ acetic acid, as illustrated in Fig. 1. Determination of elution pattern was made 
at $276 \mathrm{~nm}$ with a Beckmann Toshiba Specta 20 spectrophotometer. FI, FII and FIII were separately collected, concentrated in cellophane tubes by evaporation with fan and used for the experiments without lyophilization.

Determination of protein concentration. Nitrogen was determined by a micro-Kjeldahl method. The amount of protein was obtained by multiplying the amount of nitrogen by 5.8 .

Determination of aggregation curve. Two milliliters of $0.02 \%$ protein solution in $0.01 \mathrm{M}$ acetic acid was mixed with a equal volume of $0.2 \mathrm{M}$ sodium phosphate buffer, pH 5.6, containing $2 \mathrm{M}$ sodium chloride, and turbidity change of the reaction mixture was recorded at wave length $350 \mathrm{~nm}$ with a recording spectrophotometer. The details are described in the previous paper. ${ }^{\text {g) }}$

SDS-polyacrylamide gel electrophoresis. SDS-polyacrylamide gel electrophoresis was made in the same way as described in the previous paper. ${ }^{11)}$ For each run, $30 \mu 1$ of $0.2 \%$ protein solution dissolved in $1 \%$ sodium dodecyl sulfate (SDS) was charged.

Amino acid analysis of component polypeptides separated by $S D S$-polyacrylamide gel electrophoresis. After gel electrophoresis, protein bands were fixed with $12.5 \%$ trichloroacetic acid and taken out by cutting the gel with a razor blade. The protein was extracted with $0.1 \%$ SDS from 30 pieces of gel collected from repeated electrophoresis. After dialysis against water, the extract was dried at $40^{\circ} \mathrm{C}$ with a rotary evaporator. The hydrolysis of protein was carried out with $2 \mathrm{ml}$ of twice distilled hydrochloric acid at $110^{\circ} \mathrm{C} \pm 1^{\circ} \mathrm{C}$ for $24 \mathrm{hr}$ in a sealed and evacuated tube. The hydrolyzate was dried at $50^{\circ} \mathrm{C}$ with a rotary evaporator. For amino acid analysis, a Hitachi amino acid analyzer KLA-5 was used.

Comparison of particle size of aggregates by centrifugation. Aggregation reaction was made in the same way as described in the determination of aggregation curve, except that the volume of the protein and buffer solution used was $5 \mathrm{ml}$ for each. After $10 \mathrm{~min}$ reaction, the reaction mixture was centrifuged at a certain speed for various times with a Marusan centrifuge type 300S-1, Sakuma Seisakusho, Ltd. The centrifugation was made simultaneously among the samples compared, Supernatants were taken out with pipette after each centrifugal run and their nitrogen contents were determined by a micro-Kjeldahl method. Size of aggregating particles of protein was compared by the disappearance of nitrogen in supernatants.

\section{RESULTS AND DISCUSSION}

Polypeptide compositions of FI, FII and FIII obtained by gel filtration after cleavage of disulfide bonds of glutenin

Polypeptides obtained by reduction and cyanoethylation of glutenin were fractionated into three fractions, FI, FII and FIII, by gel filtration on a Sephadex G-150 column, as illustrated in Fig. 1. The three fractions were analyzed by SDS-polyacrylamide gel electrophoresis. Electrophoretic patterns obtained for Alberta glutenin are given in Fig. 2, where the designations of bands are made according to Danno et al. ${ }^{12)}$ with additional bands $2^{\prime}, 3^{\prime}$ and $4^{\prime}$. The electrophoretic patterns before

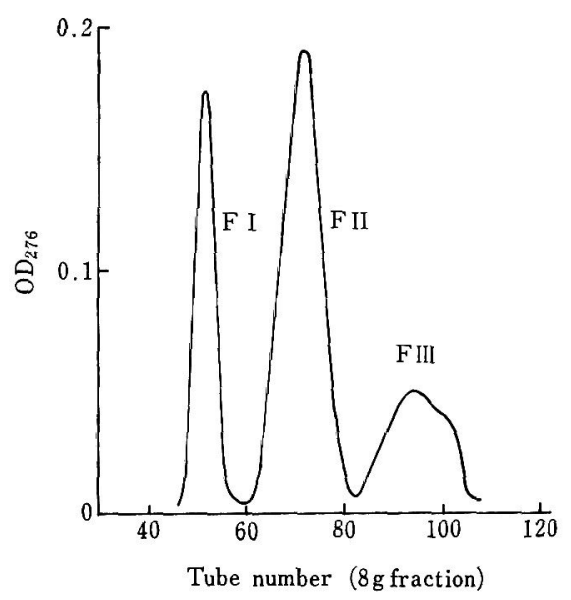

FIG. 1. Gel Filtration Pattern of Glenlea Glutenin after Cleavage of Disulfide Bonds.

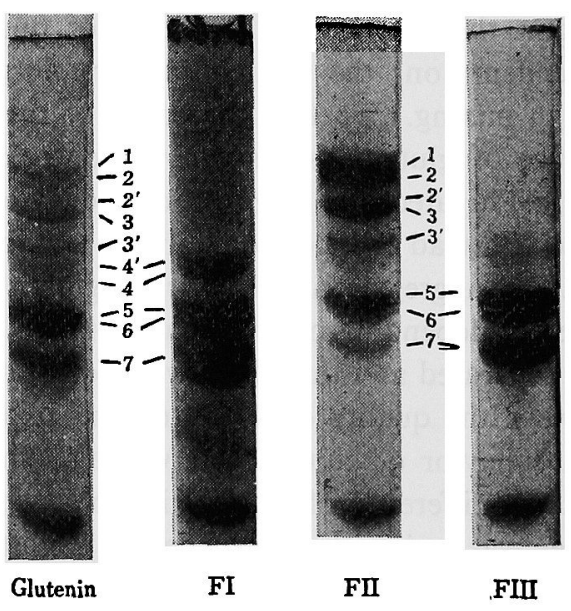

FIG. 2. SDS-Polyacrylamide Gel Electrophoresis of Component Polypeptides of Glutenin before Fractionation, FI, FII and FIII. 
fractionation showed that Sww, Sws and Thatcher lacked Band 3', and that Wakooma, a durum flour, lacked Band 1, $3^{\prime}$ and $4^{\prime}$. The lack of Band 1 and 2 for durum wheat glutenin are pointed out by Bietz and Wall, ${ }^{13}$ and Orth and Bushuk, ${ }^{14}$ ) but Band 2 was present in the case of Wakooma. As shown in Fig. 2, FI consisted of Band 4, 5, 6 and 7 and a weak band, 4'. FII consisted of Band 1, 2, 2', 3 and 5 and weak bands, 3', 6 and 7 . FIII consisted of Band 5, 6 and 7. Band 5, 6 and 7 were contained in both FI and FIII as major components. To determine whether the components of Band 5, 6 and 7 belonging to FI are identical with those belonging to FIII or not, amino acid analysis of the bands was made with Alberta glutenin. As Band 5 and 6 was too close to separate, they were combined to analyze as Band $5+6$. The results obtained are shown in Table I, combined with the results of Danno et al. ${ }^{13)}$ for Band 1, 2, 3 and 4. As shown in Table I, Band $5+6$ and 7 belonging to FI were considerably different from those belonging to FIII in the contents of glutamic acid, proline and lysine, indicating that polypeptides of Band 5, 6 and 7 belonging to FI were different from those belonging to FIII, in spite of the same mobilities in SDS-polyacrylamide gel electrophoresis.

As is seen in Table I, Band $5+6$ and 7 in FI were very similar in amino acid composition, while Band 4 in the same fraction showed considerale difference from those bands.

However, they are common in a higher content of lysine and lower contents of glutamic acid and proline in comparison with the other component polypeptides of glutenin. Band 1, 2 and 3 which were main components of FII were similar in amino acid composition, being characterized by higher contents of glutamic acid, glycine and tyrosine, and lower contents of leucine and phenylalanine. In FIII, Band $5+6$ and 7 were also similar, being higher in glutamic acid, proline and phenylalanine content and lower in glycine and tyrosine content. These results indicated that each of the three fractions consisted of a group of polypeptides which were similar or common in amino acid composition within the group.

Tarle I. Amino Acid Compositions of Polypeptides Obtained BY SDS-POLYACRYLAMIDE GEL ELECTROPHORESIS

Polypeptides were obtained from Alberta glutenin. Values shown are expressed as number of residues per 100 total residues. Tryptophane was not determined. In this experiment, the elution peak for cysteine overlaps with the glutamic acid peak, ${ }^{16)}$ but the content of cysteine is very low. ${ }^{16)}$

\begin{tabular}{|c|c|c|c|c|c|c|c|c|}
\hline \multirow{2}{*}{ Amino acid } & \multicolumn{3}{|c|}{ FI } & \multicolumn{3}{|c|}{$\mathrm{FII}^{a)}$} & \multicolumn{2}{|c|}{ FIII } \\
\hline & $4^{a)}$ & $5+6$ & 7 & 1 & 2 & 3 & $5+6$ & 7 \\
\hline Asp & 10.9 & 3.4 & $\overline{4.1}$ & 1.6 & 1.5 & 1.4 & 2.0 & 3.6 \\
\hline Thr & 1.9 & 3.0 & 4.3 & 3.5 & 3.4 & 3.6 & 3.0 & 3.1 \\
\hline Ser & 3.8 & 8.1 & 7.9 & 7.0 & 7.7 & 5.9 & 7.7 & 6.6 \\
\hline Glu & 16.6 & 27.1 & 27.3 & 36.7 & 37.8 & 37.6 & 35.1 & 30.8 \\
\hline Pro & 10.9 & 11.7 & 9.9 & 15.1 & 12.1 & 13.1 & 16.0 & 14.9 \\
\hline Gly & 8.9 & 6.2 & 6.8 & 17.2 & 17.7 & 16.9 & 4.9 & 7.0 \\
\hline Ala & 7.4 & 3.7 & 4.0 & 3.1 & 3.5 & 3.9 & 2.7 & 3.6 \\
\hline Val & 8.6 & 4.7 & 5.8 & 2.5 & 2.6 & 3.0 & 4.3 & 4.4 \\
\hline Met & 2.1 & 0.9 & 1.2 & - & - & - & 1.2 & 0.1 \\
\hline Ile & 4.1 & 3.9 & 4.2 & 1.2 & 1.0 & 1.4 & 4.4 & 4.2 \\
\hline Leu & 9.5 & 8.0 & 7.1 & 4.0 & 4.0 & 4.6 & 7.8 & 7.8 \\
\hline Tyr & 3.3 & 1.1 & 0.1 & 5.3 & 6.0 & 5.0 & 0.3 & 0.8 \\
\hline Phe & 4.3 & 4.2 & 3.3 & 0.7 & 0.7 & 1.0 & 4.7 & 4.8 \\
\hline Lys & 4.4 & 7.6 & 7.9 & 1.0 & 0.9 & 1.0 & 2.0 & 3.5 \\
\hline His & 1.0 & 3.3 & 2.9 & 0.9 & 0.8 & 0.8 & 1.6 & 2.3 \\
\hline Arg & 1.5 & 3.1 & 3.3 & 0.2 & 0.2 & 0.9 & 2.2 & 2.7 \\
\hline
\end{tabular}

a) from data of Danno et al. ${ }^{12}$ 
Aggregation behaviors of FI, FII and FIII

Aggregation behaviors of FI, FII and FIII were investigated by the turbidimetric method described in the previous paper. ${ }^{9)}$ Aggregation curves determined with the ten glutenins used in this study are shown in Fig. 3. Each of the three fractions showed its own characteristic aggregation curve, the same fractions of different sources giving closely related curves. Only exceptions were Pitic and Manitou, whose FIII gave extraordinarily high value of turbidity. The average values of $\tau_{10} / \mathrm{C}$, the turbidity at $10 \mathrm{~min}$ aggregation reaction per unit protein concentration $(C=0.01 \%)$, were 35 for FI, 90 for FII and 70 for FIII.

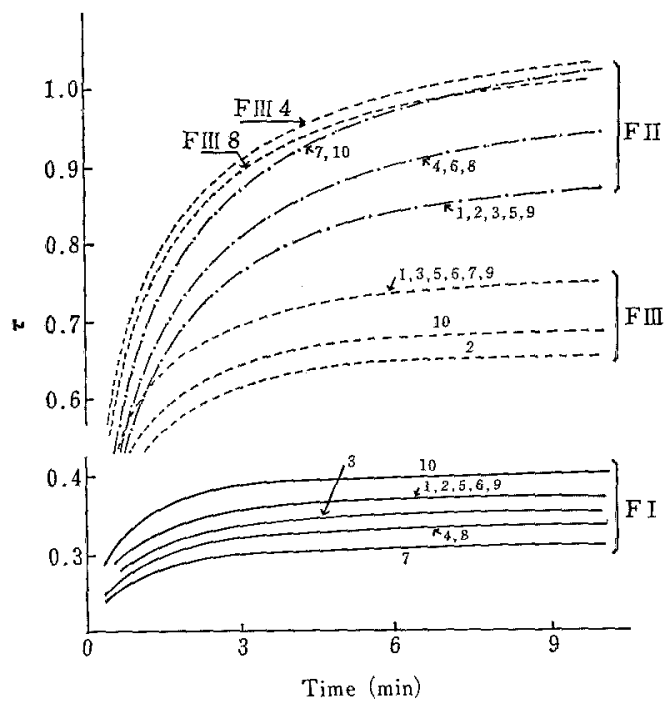

FIG. 3. Time Course of Turbidity for FI, FII and FIII Prepared from Ten Flour Glutenins.

Numbers show the kinds of original flours listed in Table I from which the fractions were prepared.

The sizes of the aggregated particles of FI, FII and FIII were compared by centrifugation at $800 \mathrm{rpm}$ with Glenlea glutenin. The results are shown in Fig. 4. The rate of sedimentation increased in the order of FII, FIII and FI, indicating that the size of aggregating particles increased in this order. $\tau_{1.0} / \mathrm{C}$ described above decreased in this order. This is against expectation, because turbidity usually increases with proceeding of aggregation. However, the turbidity decreases on the contrary, when particles grow over a certain limit, and this is the case. Thus, the low turbidity of FI throughout the aggregation curve recorded is due to the presence of large particles even in the initial stage of aggregation reaction. Such a high tendency to aggregation of FI polypeptides has been pointed out by Kanazawa and Yonezawa, ${ }^{17)}$ Huebner et $a{ }^{18)}$ and Hamauzu et al. ${ }^{29}$

In addition to the results of FI, FII and FIII, the results for original gultenin of Glenlea are also shown in Fig. 4. The results show that the three fractions formed larger aggregating particles than glutenin itself, suggesting that component polypeptides of glutenin acquire higher aggregating tendency through cleavage of disulfide bonds.

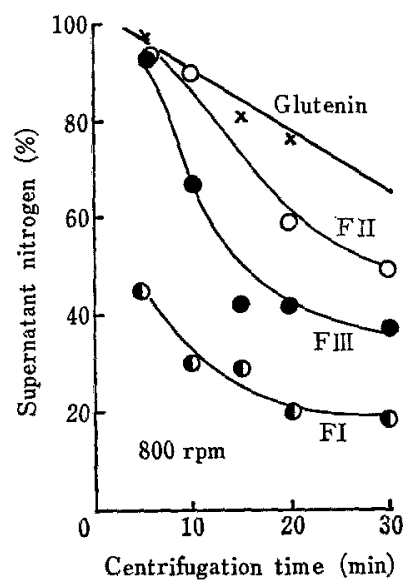

FIG. 4. Amount of Protein in Supernatant after Centrifugation in Aggregation Reaction of FI, FII and FIII.

\section{$F I, F I I$ and FIII contents of glutenins}

The amounts of FI, FII and FIII were determined after fractionation by the nitrogen determination with the ten kinds of glutenins used in this study. The results are given in Table II. The content of FIII was higher than what was expected from the peak area of gel filtration pattern (see Fig. 1). This may be attributed to the low content of tyrosine and tryptophane in FIII in comparison with FI and FII. ${ }^{19)}$ Significant difference was observed in FI, FII and FIII content among the ten glutenins. Since FI, FII and FIII were dis- 


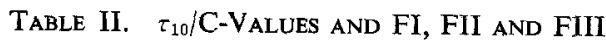
Contents of Glutenins Separated from VARIOUS Flours

\begin{tabular}{rlrrrrr}
\hline Flour & $\tau_{10} / \mathrm{C}^{a}$ & $\begin{array}{r}\text { FI } \\
\%\end{array}$ & $\begin{array}{r}\text { FII } \\
\%\end{array}$ & $\begin{array}{c}\text { FIII } \\
\%\end{array}$ & $\begin{array}{c}\text { FII: FI } \\
\text { Ratio }\end{array}$ \\
\hline 1 & Sww $^{b)}$ & 87 & 21 & 22 & 57 & 1.05 \\
2 & Sws $^{0)}$ & 89 & 20 & 20 & 60 & 1.00 \\
3 & Neepawa $^{2}$ & 89 & 18 & 26 & 56 & 1.44 \\
4 & Manitou & 90 & 18 & 26 & 56 & 1.44 \\
5 & Alberta & 97 & 17 & 27 & 56 & 1.58 \\
6 & Thatcher & 97 & 21 & 30 & 49 & 1.43 \\
7 & Wakooma & 104 & 13 & 24 & 63 & 1.85 \\
8 & Pitic & 105 & 10 & 28 & 62 & 2.80 \\
9 & Norquay & 105 & 16 & 26 & 58 & 1.63 \\
10 & Glenlea & 117 & 14 & 33 & 53 & 2.36 \\
\hline
\end{tabular}

a) Values shown were determined in the previous paper. $\tau_{10}$ : turbidity at $10 \mathrm{~min}$. C: protein concentration, which was $0.01 \%$ in all cases.

b) Soft white winter.

c) Soft white spring.

tinctive in the polypeptide composition and the aggregation behavior as described above, it might be probable to assume that the difference of aggregation behaviors of glutenins is due to the difference of FI, FII and FIII contents in glutenins.

Relation between aggregation behavior of glutenin and its FI, FII and FIII content

Relation between the aggregation behavior of glutenin and its FI, FII and FIII content was investigated by using aggregation data reported in the previous paper ${ }^{8)}$ and the FI, FII and FIII contents obtained above.

It was pointed out in the previous paper $^{8)}$ that the $\tau_{10} / \mathrm{C}$ value of glutenin markedly differed among the ten glutenins. Figure 5 shows the plot of $\tau_{10} / \mathrm{C}$ of glutenin against FI content. Though the plot showed some scatters, a tendency was observed that $\tau_{10} / \mathrm{C}$ decreased with the increasing FI content. By assuming a linear relationship between $\tau_{10} / \mathrm{C}$ and FI content, the regression line and the correlation coefficient were determined by the least squares method. The coefficient 0.726 was obtained to the regression line given in Fig. 5, indicating a considerable correlation between $\tau_{10} / \mathrm{C}$ and FI content of glutenin. In Fig. $6, \tau_{10} / \mathrm{C}$ of glutenin was plotted against

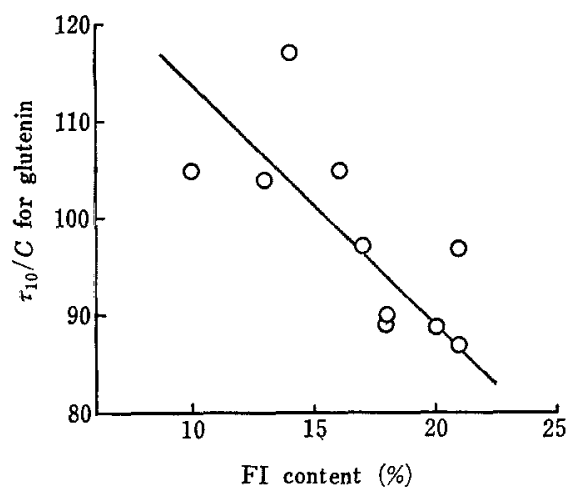

FIG. 5. Correlation between $\tau_{10} / \mathrm{C}$ and FI Content of Glutenin.

Solid line: regression line.

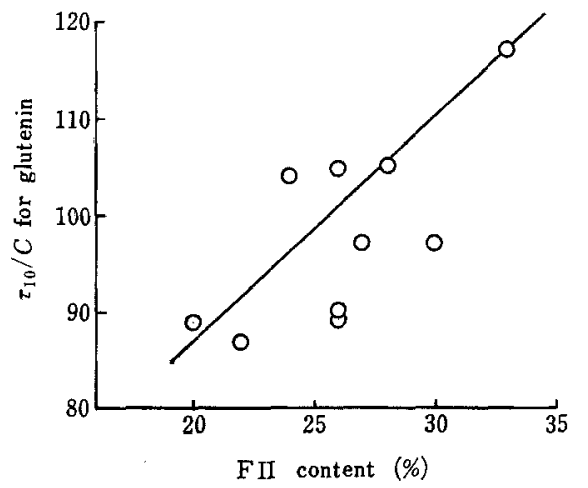

FIG. 6. Correlation between $\tau_{10} / \mathrm{C}$ and FII Content of Glutenin

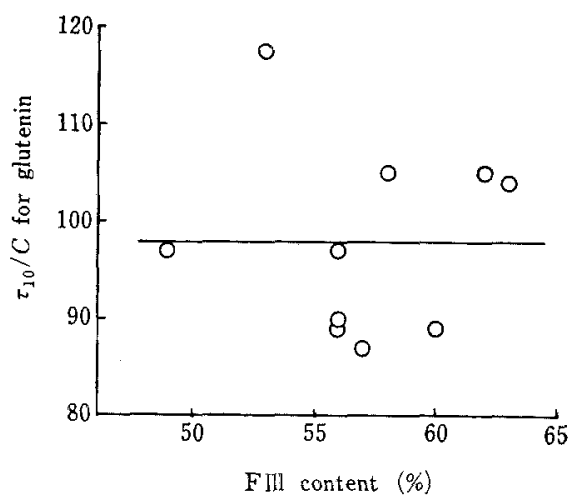

FIG. 7. Correlation between $\tau_{10} / \mathrm{C}$ and FIII Content of Glutenin.

FII content. In this case, a tendency was observed that $\tau_{10} / \mathrm{C}$ increased with increasing FII content, and the correlation coefficient obtained for this plot was 0.701 . On the 
other hand, no correlation was observed between $\tau_{10} / \mathrm{C}$ and FIII content, as shown in Fig. 7 , the correlation coefficient being close to zero. Reflecting these relations, a good correlation was obtained between $\tau_{10} / \mathrm{C}$ and FII: FI ratio of glutenin, as shown in Fig. 8, and the correlation coefficient was 0.824 . As $\tau_{10} / \mathrm{C}$ of gluten closely correlated with that of glutenin as described in the previous paper, ${ }^{8}$ similar relation can be expected for $\tau_{10} / \mathrm{C}$ of gluten. As shown in Fig. 9, relation between $\tau_{10} / \mathrm{C}$ of gluten and FII: FI ratio gave a correlation coefficient 0.760 .

The value of $\tau_{10} / \mathrm{C}$ usually increases with increasing size of aggregating particles. However, in the case of the aggregation reaction of FI polypeptides described in the previous section, the low $\tau_{10} / \mathrm{C}$ value was due to not a small but a too large particle size. Therefore,

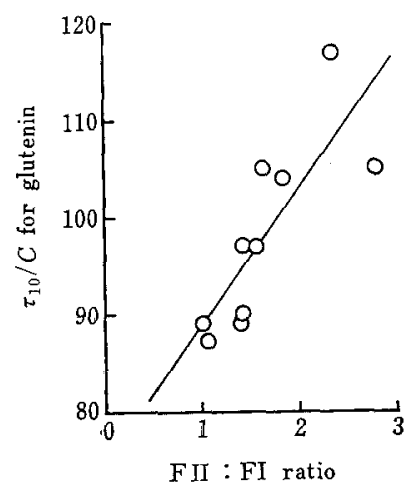

FIG, 8. Correlation between $\tau_{10} / \mathrm{C}$ and FII: FI Ratio of Glutenin.

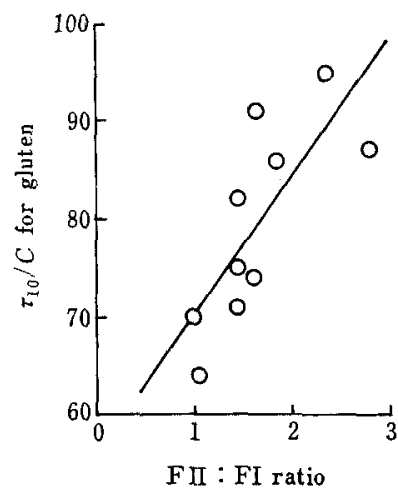

FIG. 9. Correlation between $\tau_{10} / \mathrm{C}$ of Gluten and FII: FI Ratio of Glutenin. to examine whether the low $\tau_{10} / \mathrm{C}$ value of FI-rich glutenin is also due to such a large particle size or not, the particle size of Sws glutenin, which was rich in FI and of low $\tau_{10} / \mathrm{C}$, was compared after aggregation reaction with that of Glenlea, rich in FII and of high $\tau_{10} / \mathrm{C}$, by centrifugation. The results are shown in Fig. 10. Particle size of Glenlea was much larger than that of Sws in accordance with the $\tau_{10} / \mathrm{C}$ value, as is expected in the usual case. The same relation was observed between the $\tau_{10} / \mathrm{C}$ value of gluten and its particle size, as was reported previously. " Since there is a good correlation in $\tau_{10} / \mathrm{C}$ between gluten and glutenin, it may be concluded from these results that, in the cases of gluten and glutenin, their $\tau_{10} / \mathrm{C}$ values increase with increasing size of aggregating particles.

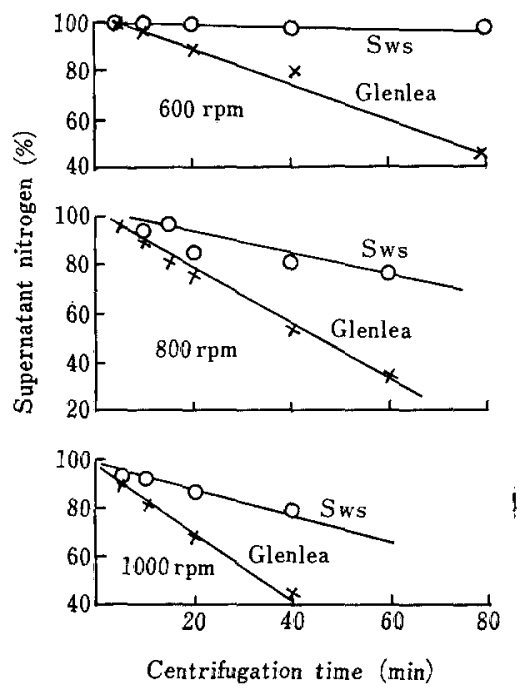

FIG. 10. Amount of Protein in Supernatant after Centrifugation in Aggregation Reaction of Sws- and Glenlea-Glutenin.

Acknowledgement. We owed much to Dr. Ibuki and Prof. Kanamori in Kyoto Prefectural University for their kind help in performing the amino acid analysis.

\section{REFERENCES}

1) M. C. Markley, Cereal Chem., 15, 438 (1938).

2) T. R. Aitken and W. F. Geddes, ibid., 15, 181 (1937). 
3) M. C. Markley, C. H. Bailey and F. L. Harrington, Cereal Chem., 16, 271 (1939).

4) T. R. Aitken and W. F. Geddes, ibid., 16, 223 (1939).

5) D. E. Smith and J. D. Mullen, ibid., 42, 275 (1965).

6) J. D. Mullen and D. E. Smith, Cereal Sci. Today, 13, 398 (1968).

7) T. Arakawa, S. Matsumoto and D. Yonezawa, Nippon Nôgeikagaku Kaishi, 48, 255 (1974).

8) T. Arakawa, H. Morishita and D. Yonezawa, Agric. Biol. Chem., 40, 1217 (1976).

9) T. Arakawa and D. Yonezawa, ibid., 39, 2123 (1975).

10) R. W. Jones, N. W. Taylor and F. R. Senti, Arch. Biochem. Biophys., 84, 363 (1959).

11) Z. Hamauzu, T. Arakawa and D. Yonezawa,
Agric. Biol. Chem., 36, 1829 (1972).

12) G. Danno, K. Kanazawa and M. Natake, ibid., 40, 739 (1976).

13) J. A. Bietz and J. S. Wall, Cereal Chem., 49, 416 (1972).

14) R. A. Orth and W. Bushuk, ibid., 50, 191 (1973).

15) J. F. Cavins and M. Friedman, J. Biol. Chem., 243, 3357 (1968).

16) F. R. Huebner, G. L. Donaldson and J. S. Wall, Cereal Chem., 51, 240 (1974).

17) H. Kanazawa and D. Yonezawa, Nippon Nógeikagaku Kaishi, 48, 113 (1974).

18) F. R. Huebner and J.S. Wall, Cereal Chem., 51, 228 (1974).

19) Z. Hamauzu, Y. Kamazuka, H. Kanazawa and D. Yonezawa, Agric. Biol. Chem., 39, 1527 (1975). 